Der Kongress tanzt 
Michael Gleich

(Hrsg.)

\section{Der Kongress tanzt}

Begeisternde Veranstaltungen,

Tagungen, Konferenzen Ein Plädoyer und Praxisbuch

Springer Gabler 
Herausgeber

Michael Gleich

Saunstorf

Deutschland

DOI 10.1007/978-3-658-04148-9

Die Deutsche Nationalbibliothek verzeichnet diese Publikation in der Deutschen Nationalbibliografie; detaillierte bibliografische Daten sind im Internet über http://dnb.d-nb.de abrufbar.

Springer Gabler

(C) Springer Fachmedien Wiesbaden 2014

Das Werk einschließlich aller seiner Teile ist urheberrechtlich geschützt. Jede Verwertung, die nicht ausdrücklich vom Urheberrechtsgesetz zugelassen ist, bedarf der vorherigen Zustimmung des Verlags. Das gilt insbesondere für Vervielfältigungen, Bearbeitungen, Übersetzungen, Mikroverfilmungen und die Einspeicherung und Verarbeitung in elektronischen Systemen.

Die Wiedergabe von Gebrauchsnamen, Handelsnamen, Warenbezeichnungen usw. in diesem Werk berechtigt auch ohne besondere Kennzeichnung nicht zu der Annahme, dass solche Namen im Sinne der Warenzeichen- und Markenschutz-Gesetzgebung als frei zu betrachten wären und daher von jedermann benutzt werden dürften.

Lektorat: Juliane Wagner, Eva-Maria Fürst

Covermotiv: () der kongress tanzt

Gedruckt auf säurefreiem und chlorfrei gebleichtem Papier

Springer Gabler ist eine Marke von Springer DE. Springer DE ist Teil der Fachverlagsgruppe Springer Science+Business Media

www.springer-gabler.de 


\section{Plädoyer für Veranstaltungen, die begeistern und bewegen}

In Deutschland nehmen jedes Jahr mehr als 300 Mio. Menschen an einer größeren Veranstaltung teil. Kongresse, Tagungen, Meetings, Foren und Events: eine Branche mit gigantischen Umsätzen. Sie gerät jedoch immer mehr unter Druck, auch durch internationale Konkurrenz. Viele Teilnehmer sind frustriert von Frontalunterricht, Nullsummenquasselei und Einpauken mit Powerpoint, von Reihenbestuhlung und Keynote-Kauderwelsch. Denn Kongresse sind Mikrokosmen, die unsere Gesellschaft spiegeln. Generation Facebook dürstet es nach Teilhabe, Einmischung, nach innovativen Stil- und Spielformen. Die Wissensgesellschaft braucht Orte für kreatives Lernen. Schließlich verlangt eine globalisierten Wirtschaft nach Vernetzung vielfältiger Perspektiven und unterschiedlicher Kulturen.

In dieser Situation entstand 2010 das „Netzwerk für gute Veranstaltungen“. Seine Mitglieder sind Moderatoren, Journalistinnen und Redner-Coaches, Kommunikationsdesignerinnen und Graphic Recorder, und ein Pastor ist auch dabei. Ein Potpourri der Perspektiven und Kompetenzen. Allen gemeinsam ist die langjährige Berufserfahrung bei der Gestaltung von Veranstaltungen. Und eine Grundhaltung, die sie in ihrem Manifest formulierten: „Wirtschaft und Gesellschaft brauchen Orte, an denen Menschen in Bewegung kommen."

In diesem Buch entwerfen sie eine mitreißende Vision: Veranstaltungen, die uns begeistern, bewegen und inspirieren. Als Räume für Potenzialentfaltung. Als freudige Lernorte für erwachsene Menschen. Unter Einbeziehung der Weisheit der Vielen. Die Autoren stützen sich auf neueste Erkenntnisse aus Hirnforschung, Kommunikationswissenschaft, Werbepsychologie und Reformpädagogik, übertragen gesellschaftliche Diskurse über Partizipation, Inklusion und kulturelle Vielfalt auf den Veranstaltungssektor.

Ihr Entwurf hat wenig mit Technik und viel mit innerer Haltung zu tun. Und mit dem Mut, sich aus Routinen zu lösen. Die Autoren nennen Beispiele erfolgreicher und außergewöhnlicher Veranstaltungen aus eigener Erfahrung, die zeigen: So bringt man Kongresse zum Tanzen. 


\section{Inhaltsverzeichnis}

\section{Teil I Ouvertüre (im Sitzen)}

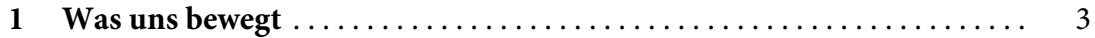

Michael Gleich, Gerriet Danz, Tina Gadow, Hans-Joachim Gögl,

Thomas Klug, Heike Leitschuh, Christian Maier, Aimé Sans,

Gabriele Schlipf und Heiner Wember

2 Von kongrässlich zu kongenial $\ldots \ldots \ldots \ldots \ldots \ldots \ldots \ldots \ldots \ldots$

Michael Gleich

Der Kongress der Zukunft $\ldots \ldots \ldots \ldots \ldots \ldots \ldots \ldots \ldots \ldots \ldots \ldots . \quad 7$

Jetzt ist Tango ................................... 9

Powerpoint und Nullsummenquasselei $\ldots \ldots \ldots \ldots \ldots \ldots \ldots \ldots \ldots, 11$

Stell dir vor, es ist Kongress, und keiner geht hin .............. 12

Ein Schwachpunkt namens Powerpoint .................... 15

Klares Ziel, spannende Dramaturgie ........................ 17

Machtspiele im Kontrollraum .......................... 19

\section{Teil II Choreografien: Vivace con moto}

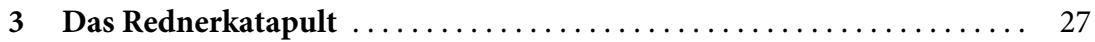

Gerriet Danz

Verbretterte Wirkung? ............................. 27

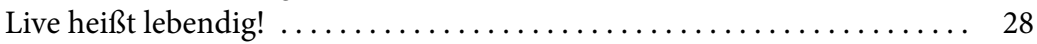

Slideshows: Klick-Klick trifft Ohren-auf-Durchzug.............. 28

Pool statt Pult .................................... 29

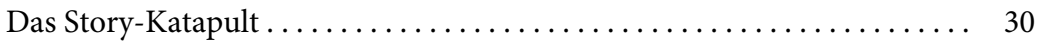

Das News-Katapult............................... 30

Das Metapher-Katapult ........................... 31 


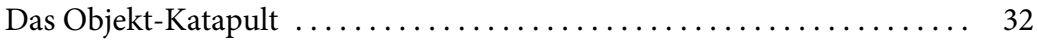

Das Demo-Katapult . ......................... 32

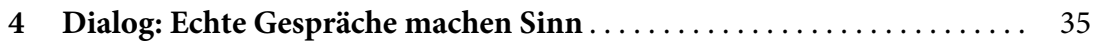

Thomas Klug und Michael Gleich

5 Das einzige was stört, ist die Zeit vor und nach der Pause . . . . . . 45

Hans-Joachim Gögl

Der sprachlose Patient ....................... 46

Zurück von der Front zum Frontalunterricht $\ldots \ldots \ldots \ldots \ldots \ldots \ldots \ldots$

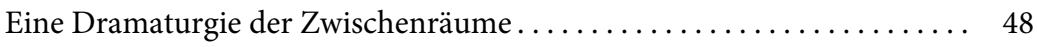

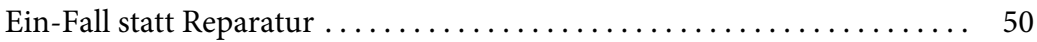

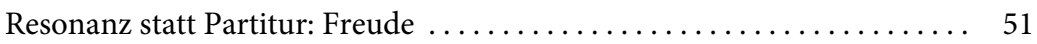

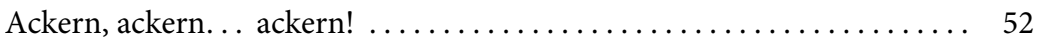

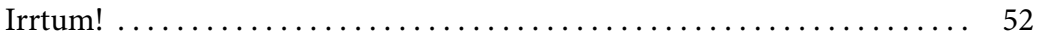

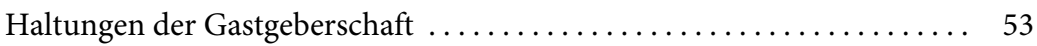

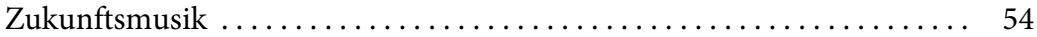

Eine Murmelpause, ein Gasthaus und dann gute Nacht . . . . . . . 55

Zuhören üben: Selbstvergessen, wach und verbunden $\ldots \ldots \ldots \ldots \ldots . . \ldots 5$

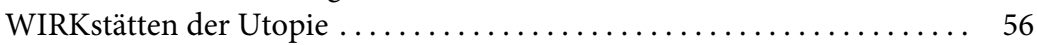

Die Leere auf der Landebahn für Ein- und Zufälle . . . . . . . . . . . 57

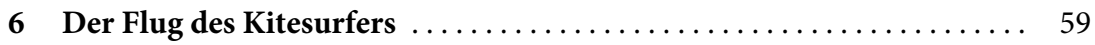

Tina Gadow

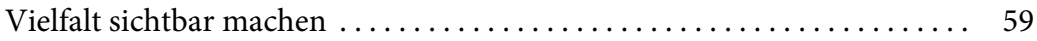

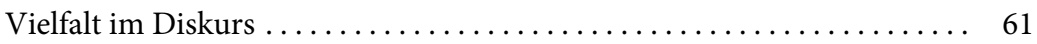

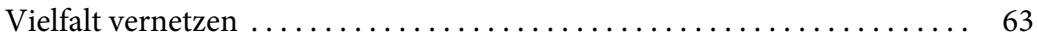

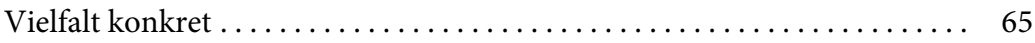

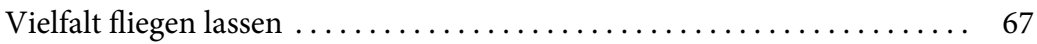

$7 \quad$ 1648: Prahlen, drohen, schmieren ................... 69

Heiner Wember

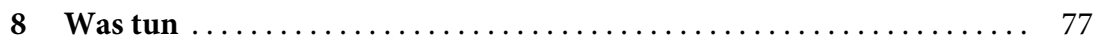

Aimé Sans

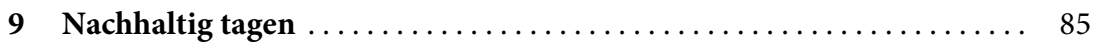

Heike Leitschuh

Nachhaltigkeit erfordert fundamentale Veränderungsprozesse . . . . . . 86

Alternativen, Emotionen und Vorbilder ................ 87 
Stimmige Kongresse $\ldots \ldots \ldots \ldots \ldots \ldots \ldots \ldots \ldots \ldots \ldots \ldots \ldots$

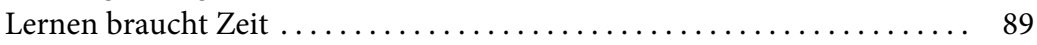

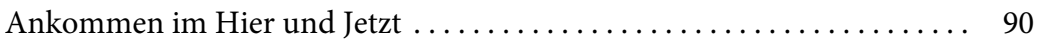

Auch Experten können etwas lernen $\ldots \ldots \ldots \ldots \ldots \ldots \ldots \ldots \ldots \ldots \ldots$

Innovative Formate sind auch nur eine Hülle $\ldots \ldots \ldots \ldots \ldots \ldots \ldots . \ldots 2$

Kontraproduktiver Fleiß ........................ 93

Geist, Körper und Psyche ansprechen $\ldots \ldots \ldots \ldots \ldots \ldots \ldots \ldots . \ldots \ldots$

Konsistenz oder: Veranstalten, was man predigt ............... 94

Theorie und Praxis in spannender Choreografie .............. 96

\section{Teil III Pirouette und Révérence}

10 Sicherheit im Umgang mit Unsicherheit . . . . . . . . . . . . . . . . 99

Christian Maier

11 Chaordische Kongresse . . . . . . . . . . . . . . . . . . 107

Michael Gleich

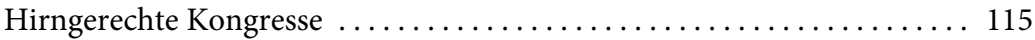

Das äußere Format ist nichts, die innere Haltung alles . . . . . . . . 120

Lebendigkeit zwischen Chaos und Ordnung ............... 122

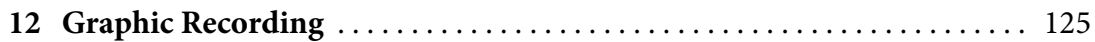

Gabriele Schlipf

GRAPHIC RECORDING - Visuelles Protokoll //live und simultan . . . . 125

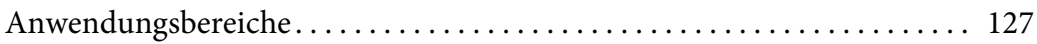

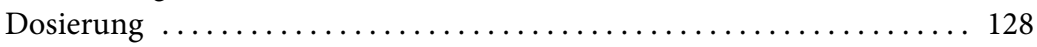

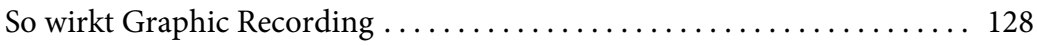

Aufbewahrung des visuellen Protokolls . . . . . . . . . . . . . . . . . 129 


\section{Mitarbeiterverzeichnis}

Gerriet Danz praesentarium, Siemssenstraße 3, 20251 Hamburg, Deutschland E-mail:danz@praesentarium.com

Tina Gadow Vielfalt gestalten, Senefelderstr. 28, 10437 Berlin, Deutschland E-mail: post@tinagadow.de

Michael Gleich der story teller, Alte Dorfstraße 8, 23996 Saunstorf, Deutschland E-mail: michaelgleich@t-online.de

Hans-Joachim Gögl Hans-Joachim Gögl Strategie und Kommunikation GmbH, Belruptstrasse 17, 6900 Bregenz, Österreich

E-mail: hansjoachim@goegl.com

Thomas Klug cogitamus, Fritz-Bell-Str. 4, 61239 Ober-Mörlen, Deutschland E-mail: thomas.klug@cogitamus.eu

Heike Leitschuh Fair Wirtschaften, Münchener Str. 23, 60329 Frankfurt a. M., Deutschland

E-mail: heike-leitschuh@t-online.de

Christian Maier inner game Institut, Klosterwaldstr. 13, 79295 Sulzburg, Deutschland

E-mail: maier@innergame.de

Aimé Sans BUSINESS \& NATURE GmbH, Rotwandstrasse 26, 81539 München, Deutschland

E-mail: asans@business-nature.de 
Gabriele Schlipf Manufaktur für visuelle Kommunikation - momik*, Berlin, Deutschland

E-mail: gabriele@momik.de

Heiner Wember AUF DEN PUNKT, Lütkenbecker Weg 100, 48155 Münster, Deutschland

E-mail: HeinerWember@t-online.de 


\section{Die Autoren}

Gerriet Danz Jahrgang 1965, konzipiert und inszeniert Vorträge, die auf kreative Weise begeistern. Er erarbeitet Botschaften, die verstanden werden. Und er trainiert Vortragende, die ihre Inhalte in den Köpfen und Bäuchen ihres Publikums langfristig verankern möchten. Heute einer der anerkanntesten Kommunikationstrainer Deutschlands, startete Danz seine Karriere als Kreativdirektor der internationalen Werbeagentur BBDO. 1995 wird er vom damaligen Spiegel-Chefredakteur Stefan Aust fürs Fernsehen entdeckt und moderiert die VOX News sowie 220 Folgen des Wissens-Show- Klassikers JEOPARDY. 2010 erscheint bei CAMPUS sein Buch „Neu präsentieren“, das bereits nach drei Wochen Platz 1 der RatgeberBestsellerliste bei Amazon eroberte. Gerriet Danz ist Mitglied der GSA German Speakers Association, der Top 100 Speaker bei Speakers Excellence und Preisträger des CONGA Awards in der Kategorie „Bester Referent \& Trainer“.

Tina Gadow Jahrgang1975, studierte Theaterwissenschaft und kulturelle Kommunikation in Paris und Berlin. Nach dem Studium tauchte sie in die französische Theater- und Festivalwelt ein und begleitete internationale Austauschprogramme. Sie arbeitete zunächst im Hauptstadtbüro des Goethe-Instituts und war anschließend vier Jahre lang für den Schwerpunkt ,Kultur und Entwicklung' im Büro Berlin der GTZ zuständig. Kern ihrer Arbeit war immer die Auseinandersetzung mit gesellschaftlichen und (kultur-) politischen Themen im Rahmen von Wortveranstaltungen. Der Wunsch danach, mehr Kreativität und Vergnügen in diese Begegnungen zu bringen, dabei unterschiedliche Ansätze, Entwürfe und Perspektiven zusammen zu führen und mit Akteuren aus Zivilgesellschaft, Politik und Kultur gleichermaßen eng zusammen zu arbeiten führte sie in die Selbständigkeit: Unter dem Motto „Vielfalt gestalten“ gründete sie $2008 \mathrm{ihr}$ eigenes Büro in Berlin. Seitdem konzipiert, berät, begleitet und moderiert sie internationale und lokale Konferenzen, Workshops, Salons, Netzwerktreffen. Als Chansonnière mag sie die Bühne. 
Michael Gleich Jahrgang 1960, hat lange Zeit als Reporter für Magazine wie GEO, stern und natur gearbeitet. Bücher u. a. „Mobilität. Warum sich alle Welt bewegt“; „Life Counts - Eine globale Bilanz des Lebens“; „Web of Life. Die Kunst vernetzt zu leben“; „Wir kündigen. Und definieren das Land neu“; „Die Friedensmacher“; „Culture Counts. Wie wir die Chancen kultureller Vielfalt nutzen“. Er gehört zu den am häufigsten ausgezeichneten Journalisten in Deutschland, unter anderem: Wissenschaftsbuch des Jahres, Buchpreis der Deutschen Umweltstiftung, und zweimal mit dem Medienpreis Entwicklungspolitik, verliehen vom Bundespräsidenten. Anfang der Neunziger Jahre begann er, Unternehmen im Bereich Kommunikation und Nachhaltigkeit zu beraten. Immer öfter fand er sich als Moderator von Podiumsdiskussionen auf der Bühne wieder. Oder als Vortragsredner bei Konferenzen. Zusammen mit seiner Kollegin Tina Gadow gründete er das Netzwerk „der kongress tanzt" und koordiniert dessen Aktivitäten.

Hans-Joachim Gögl Jahrgang 1968, erstes Zeitungsprojekt mit zwölf, Buchhändlerlehre mangels Gymnasiumstauglichkeit, danach fester freier Mitarbeiter in der Radiokulturredaktion des ORF - Features, Portraits, Literaturbesprechungen für das Landesstudio Vorarlberg und Ö1. 1992 Eröffnung eines Büros für Öffentlichkeitsarbeit. Bis heute Entwicklung und Projektleitung von Kommunikationsstrategien für öffentliche und private Auftraggeber, Konzeption und Programmleitung von Tagungen/Kongressen/Festivals, Begleitung von regionalen Entwicklungsprozessen im Bereich Kultur. Diverse Auszeichnungen, darunter Träger des Österreichischen Staatspreis für Erwachsenenbildung 2011, sowie Staatspreis-Nominierungen in den Bereichen Öffentlichkeitsarbeit 1999 und Marketing 2004. Seit 2011 Dozent an der Universität Graz zum Thema Marketing und Öffentlichkeitsarbeit im Bereich Pflegemanagement.

Thomas Klug Jahrgang 1960, Lehre zum Industriekaufmann, Studium der Betriebs-, Volkswirtschaft und Philosophie in Tübingen und Münster, Diplom Kaufmann, Langjährige Berufserfahrung in Industrie (Krups und Continental) und Beratung (Kienbaum, PA Consulting und IMIG), internationaler Projektleiter in Ost -und Westeuropa sowie Ostafrika, Personal- und Changemanager, Marketingund Vertriebsmanager, Geschäftsführer von Vertriebsgesellschaften in Südafrika und USA, Akademieleiter Gründer und Geschäftsführender Partner von cogitamus, consulting-training-coaching. Arbeitsschwerpunkte: Consulting, Training und Coaching zu den Themenfeldern Führung, Transformation, Kaizen und Lean Administration, Organisation und Innovation.

Dozententätigkeit: Alanus Hochschule, Alfter/Bonn; Karlshochschule, Karlsruhe; Provadis School of International Management and Technology AG, Frankfurt a. M.; Steinbeis Business Academy, Gernsbach. 
Heike Leitschuh Jahrgang 1958, Studium der Politischen Wissenschaften, Soziologie, Volkswirtschaft, Abschluss als Diplom-Politologin. Bis 1995 Redakteurin beim Fachverlag Ökologische Briefe in Frankfurt a. M., dort Aufbau und Leitung des Ressorts „Umwelt und Wirtschaft“. Seit 1995 Tätigkeit als selbständige (Buch-) Autorin, Moderatorin und Beraterin. Seit Ende 2001 Mitherausgeberin des Jahrbuch Ökologie, C.H. Beck-Verlag, seit 2008 im Hirzel-Verlag, Stuttgart. Breites Netzwerk von Autor/innen, Wissenschaftler/innen und Berater/innen mit zusätzlichem Know-how und Fähigkeiten. Fachliche Expertise zu CSR, nachhaltigem Wirtschaften und nachhaltigen Lebensstilen, Postwachstumsökonomie. Bücher u. a. „Nachhaltig die Zukunft managen. Pioniere in globalen Unternehmen. Porträts und Hintergründe“, Bern 2005, „Lust auf Stadt. Ideen und Konzepte für urbane Mobilität“, Bern 2002.

Christian Maier Jahrgang 1957. Nach dem BWL-Studium in Frankfurt a. M., Paris und London baute er einen Verlag auf und konzipierte, produzierte und vertrieb Lernmaterialien für leichtes und spielerisches Lernen (Superlearning). Es folgte eine Zeit als Berater und Trainer, die schließlich in der Gründung des „Inner Game Institutes" mündete. Seitdem versteht er sich als Wegbegleiter von Unternehmen, Führungskräften, Trainern und Coaches. Eine seiner Ansätze dabei ist WanderCoaching. Teilnehmer seiner Seminare und Weiterbildungen bezeichnen ihn als einen unkonventionellen Menschen mit sehr viel Phantasie, einen Lernkünstler und Lernakrobaten, der Systeme und sich darin bewegende Menschen mit viel Feingefühl an hinderliche Grenzen heranführt. Diese Fähigkeit macht ihn zu einem gefragten „Spielraumgestalter“. Christian Maier versteht es, Menschen dazu zu bewegen, sich selbst zu bewegen.

Aimé Sans Jahrgang 1961, Mitbegründer und Geschäftsführer der Agentur „Business \& Nature“ in München. Er sagt über seine Arbeit: „Event-Kompetenz und das Beherrschen von unterschiedlichen Trainingsmethoden sind unsere Werkzeuge für erfolgreiche, dialogorientierte Veranstaltungen.“

Gabriele Schlipf Jahrgang 1973. Nach dem Studium der visuellen Kommunikation an der HfG Schwäbisch Gmünd und FH Düsseldorf arbeitete sie über zehn Jahre als freiberufliche Creative-Direktorin. Sie gestaltete nach dem Grundsatz „Design based upon concepts" und wendete diesen hauptsächlich auf Corporate Design und Informationsgestaltung an. Seit 2011 arbeitet sie überwiegend als Graphic Recorder. Sie versteht sich als zeichnende Simultan-Übersetzerin von gesprochenem Wort in sichtbare Bilder. Sie protokolliert für Wirtschaft, Wissenschaft, Politik und Kultur jegliches Thema bei Kongressen, Diskussionen, Workshops oder Vorträgen im Inund Ausland. Die Gruppengrößen reichen von drei bis 900 Personen. 
Heiner Wember Jahrgang 1959. Versteht sich darauf, Kompliziertes einfach und spannend zu erklären. Als Hörfunk- und Fernsehjournalist erklärt der promovierte Historiker einem Millionenpublikum mit einfachen Worten schwierige Sachverhalte: Wie die Ammoniak-Synthese funktioniert, warum die Vandalen Opfer von Rufmord wurden, was einen guten Feuerlöscher ausmacht. Wember ist regelmäßig als Dozent unterwegs und weiß sein Publikum zu begeistern. Er bringt Menschen bei, auf den Punkt zu kommen: beim Reden, Schreiben und Präsentieren. 